This item was submitted to Loughborough's Research Repository by the author.

Items in Figshare are protected by copyright, with all rights reserved, unless otherwise indicated.

\title{
Integrative Verhandlungen fördern - ein experimenteller Ansatz zur Nutzung farbiger Beleuchtung in Verhandlungssituationen
}

PLEASE CITE THE PUBLISHED VERSION

https://doi.org/10.1002/bapi.201710021

\section{PUBLISHER}

Wiley (c) Ernst \& Sohn Verlag für Architektur und technische Wissenschaften GmbH \& Co. KG

\section{VERSION}

AM (Accepted Manuscript)

\section{PUBLISHER STATEMENT}

This work is made available according to the conditions of the Creative Commons Attribution-NonCommercialNoDerivatives 4.0 International (CC BY-NC-ND 4.0) licence. Full details of this licence are available at: https://creativecommons.org/licenses/by-nc-nd/4.0/

\section{LICENCE}

CC BY-NC-ND 4.0

\section{REPOSITORY RECORD}

Kombeiz, Olga, and Anna Steidle. 2019. "Integrative Verhandlungen Fördern - Ein Experimenteller Ansatz Zur Nutzung Farbiger Beleuchtung in Verhandlungssituationen”. figshare. https://hdl.handle.net/2134/37588. 
Integrative Verhandlungen fördern - ein experimenteller Ansatz zur Nutzung farbiger Beleuchtung in Verhandlungssituationen

Olga Kombeiz $z^{1}$ und Anna Steidle $e^{2}$

Promotionskolleg „,Menschen in Räumen“(MIR)

${ }^{1}$ Fachgebiet Wirtschafts- und Organisationspsychologie, Universität Hohenheim

${ }^{2}$ Management und Recht, Hochschule für Verwaltung und Finanzen Ludwigsburg

Manuscript accepted for publication in

Bauphysik [Building Physics] - February 2017

The manuscript may not exactly replicate the final version published in the journal. It is not the copy of record. The final article will be available, upon publication, via its DOI:

Kombeiz, O. \& Steidle, A. (2017). Integrative Verhandlungen fördern-ein experimenteller Ansatz zur Nutzung farbiger Beleuchtung in Verhandlungssituationen [Promoting integrative negotiations-an experimental approach for using colored lighting in negotiation situations]. Bauphysik, 39 (3), 197-201. https://doi.org/10.1002/bapi.201710021 
Das Verhandlungsgeschick der Mitarbeiter trägt in vielen Organisationen wesentlich zum Unternehmenserfolg bei. Gleichzeit weist aktuelle Forschung daraufhin, dass zwischenmenschliches Verhalten zum Beispiel in Verhandlungen durch die physikalische Umgebung beeinflusst werden kann. Die vorliegende Studie untersuchte die Bedeutung des farbigen Lichts für kooperatives Verhalten in integrativen Verhandlungen. Wir nahmen an, dass rotes Licht eine angenehme, sozialfreundliche Raumatmosphäre schafft und somit den Verhandlungserfolg fördert. In einer experimentellen Studie wurde die Lichtfarbe (rot vs. blau und weiß) variiert. Die Ergebnisse bestätigten unsere Annahme. Der theoretische Wert, Limitationen sowie praktische Implikationen der Befunde werden diskutiert.

\section{Promoting integrative negotiations - an experimental approach for using colored lighting in} negotiation situations. Employees' negotiating skills substantially contribute to organizational success. In this context, recent research shows that the physical environment may influence interpersonal behavior for example in negations. The present study investigated the importance of colored light for cooperative behavior in integrative negotiations. We assumed that red light creates a pleasant, socially friendly atmosphere and thus promotes negotiation success. In an experimental study, the light color (red vs. blue and white) was varied. The results confirmed our assumption. The theoretical value, limitations and practical implications of the findings are discussed. 


\section{Einleitung}

Wie die physikalische Umgebung den Nutzer in seinen Aktivitäten optimal unterstützen kann, entwickelt sich zu einem wichtigen Thema in der Arbeitsforschung [1]. In diesem Kontext rückt die Bedeutung von Lichtsituationen in Räumen in den Fokus, da Menschen etwa 80 bis 90 $\%$ der Informationen aus der Umgebung auf dem visuellen Weg aufnehmen [2]. Um jedoch konkrete Lösungen für eine leistungsförderliche Gestaltung der Beleuchtung anbieten zu können, ist die Berücksichtigung der Aufgabenart nötig, die im jeweiligen Raum und im Rahmen jeweiliger Tätigkeit ausgeübt wird. Aktuelle Forschung deutet nämlich darauf hin, dass je nach Aufgabenart unterschiedliche Lichtsituationen optimal sein können [1]. Die bestehenden bauphysikalischen Normen [3, 4] liefern zwar die Mindestanforderungen an die Beleuchtungsverhältnisse, beziehen jedoch die psychologische Wirkung des Lichts auf die Leistung oder Verhalten nur in geringerem Maße ein. Dabei zielen die definierten Mindestanforderungen in erster Linie auf die Ebene des physischen Komforts ab, nicht aber auf eine optimale Passung zwischen Raumbedingungen und den emotionalen und kognitiven Erfordernissen einer Arbeitstätigkeit [5]. Beispielsweise erweist sich helles kaltes Licht als förderlich für konzentriertes Arbeiten [6, 7]. Die gleiche Beleuchtungssituation würde für ein informelles Gespräch allerdings eine kühle und formelle Raumatmosphäre erzeugen und somit die Vertraulichkeit behindern.

Ähnlich zeigt die aktuelle Forschung, dass je nach Aufgabenart unterschiedliche visuelle Attribute förderlich sein können. So fand eine Reihe von Studien, dass kreative Leistung im gedimmten Licht steigt [8], während visuelle Leistung von hellem Licht profitiert [9]. Ähnliche Ergebnisse zeigen sich bei Farbstudien [10, 11]. Hier ist allerdings eine hohe Kontextsensitivität der Farbwirkung zu beachten [12]. So kann beispielsweise rote Objektfarbe (z.B. eine rote Arbeitsmappe) zu höherer oder vermindernder kreativer Leistung führen, in Abhängigkeit davon, ob die Leistungssituation erfolgsversprechend ist oder nicht [13]. Grund für diese Leistungsveränderungen sind automatische Assoziationen zwischen bestimmten Umweltstimuli (Beleuchtungssituationen, Farben) und psychologischen Prozessen, die bei der Wahrnehmung der Umweltstimuli automatisch ausgelöst werden [14]. Um diesen Zusammenhang zwischen konkreten physikalischen Stimuli (bspw. physische Wärme) und den abstrakten Konstrukten (bspw. soziale Wärme) tiefer zu verstehen, ist der situative Kontext bzw. die Aufgabenart zu berücksichtigen [15]. Während in einer Arbeitssituation Wissensarbeit als Einzelleistung erbracht werden muss, löst sie bestimmte psychologische Prozesse bei einer Person aus. In einer sozialen Situation sind dagegen mindestens zwei Personen beteiligt. Daher spielt hier die gesamte Raumatmosphäre eine Rolle, die das Entstehen von Verbundenheit zwischen den Beteiligten fördern oder behindern kann [15]. 
Soziale Situationen stellen ein zentrales Element zahlreicher berufsrelevanter Aktivitäten dar. Hierzu zählen alle Situationen, in welchen soziale Interaktion stattfindet wie beispielsweise im Verkauf, bei der Teamarbeit, in Meetings, während Pausen und in Verhandlungssituation. Insbesondere in Verhandlungssituationen steht für Unternehmen viel auf dem Spiel. Für den Erfolg und die Wettbewerbsfähigkeit von Unternehmen ist daher entscheidend, dass ihre Mitarbeiter in Verhandlungssituationen effektiv und lösungsorientiert vorgehen. Ein optimales Verhandlungsergebnis stellt eine integrative Lösung dar, das die Interessen aller Verhandlungspartner berücksichtigt. Eine solche Lösungsfindung erfordert es, dass alle Verhandlungspartner miteinander kooperieren. Daher stellt sich für die Arbeitsforschung die Frage, welche räumlichen Bedingungen und insbesondere welche Lichtbedingungen ein kooperatives Vorgehen bei integrativen Verhandlungen wahrscheinlicher machen.

\section{Das Licht in sozialen Situationen}

Um die Lichtwirkungen in sozialen Situationen zu verstehen, ist es wichtig, die Raumatmosphäre zu betrachten, die das jeweilige Licht kreiert. Eine durch das Licht erzeugte gemütliche und ungezwungene Raumatmosphäre $[16,17]$ kann soziale Nähe fördern und zur Kooperation anregen [18]. So zeigt eine Reihe von Studien, dass Licht die soziale Wahrnehmung und das soziale Verhalten beeinflussen kann [18, 19, 20]. Dieser Effekt lässt sich dadurch erklären, dass bestimmte Lichtbedingungen eine Atmosphäre schaffen, die soziale Interaktionen erleichtern. In ähnlicher Weise zeigt sich, dass angenehme Lichtbedingungen eine Zufriedenheit hervorrufen kann, welche sich positiv auf die Wahrnehmung anderer Personen auswirkt [15].

Eine gemütliche und gute Stimmung erzeugende Raumatmosphäre kann geschaffen werden, indem rötliches Licht eingesetzt wird [16]. Die automatische Assoziation des warmen rötlichen Lichts mit einem gemütlichen Wohnzimmer sendet eine visuelle Botschaft der sozialen Wärme, eine Einladung, sich mit anderen verbunden zu fühlen und zu kooperieren. Bestätigend zeigt eine Studie, dass die Interpretation von Gesprächssituationen im roten (im Vergleich zu weißem) Licht eine positive Konnotation hat [21]. Die Studienteilnehmer schrieben den Gesprächspartnern höhere Freundlichkeit und gegenseitige positive Einstellungen zu. Diese Befunde deuten darauf hin, dass auch zuvorkommendes und kooperatives Verhalten durch das rote Licht gesteigert werden könnte.

In Bezug auf integrative Verhandlungen gibt es bisher allerdings wenig Forschung, die die Wirkung des farbigen Lichts untersucht. Die Bedeutung des farbigen Lichts auf psychologische Prozesse und Verhalten ist dabei nicht zu bestreiten, denn als ein gemeinsamer Faktor von Licht und Farbe übt das farbige Licht seine Wirkung auf verschiedenen Ebenen aus [9, 12]. Als Licht 
kreiert es eine Raumatmosphäre, die für bestimmte Aktivitäten unterstützend sein kann [2]. Durch die hohe Intensität, die Farbe dem Licht verleiht, wird die Raumatmosphäre in ihren positiven oder negativen Eigenschaften bestärkt [16]. Diese durch die Lichtsituation geschaffene Raumatmosphäre [17] beeinflusst die Erwartung darüber, welches Verhalten in der gegebenen Situation angemessen ist $[15,17]$. Beispielsweise hängt eine gemütliche Raumatmosphäre mit der Erwartung zusammen, dass Vertraulichkeit zwischen zwei oder mehreren Personen angebracht ist, während eine formelle Raumatmosphäre mit höherer zwischenmenschlicher Distanz einhergeht.

\section{Hypothesen und die experimentelle Studie}

Aufgrund früherer Studien zur Raumatmosphäre nahmen wir an, dass rotes Licht im Vergleich zu blauem und weißem Licht eine für soziale Aktivitäten passende Raumatmosphäre kreieren sollte. Eine solche Raumatmosphäre kann als gemütlich, lebendig und informell beschrieben werden. Basierend auf der Idee, dass rotes Licht die visuelle Botschaft sozialer Wärme sendet, wurde in der vorliegenden Studie angenommen, dass rotes Licht im Vergleich zu blauem und weißem Licht integrative Lösungen in „face-to-face“ Verhandlungen fördert. Ein Indikator für die erfolgreiche integrative Verhandlung ist das Erlangen eines gemeinsamen Nutzens [22], der auf eine starke Kooperation zwischen den Verhandlungspartnern hindeutet.

In einer Laborstudie wurden Lichtbedingungen mit rotem, blauem und weißem Licht erzeugt. Da die Studie im Rahmen eines größeren Forschungsprojektes stattfand, gab es jeweils zwei Lichtbedingungen mit farbigem Licht: jeweils eine Lichtbedingung mit direktem und eine mit indirektem Licht (Bild 1, Tabelle 1). Für die Datenauswertung wurden die beiden Bedingungen mit farbigem Licht in der vorliegenden Studie nur hinsichtlich der Lichtfarbe untersucht. Die Lichtbedingung mit dem weißem Licht enthielt etwa 50\% direktes und 50\% indirektes Licht. Zusammengefasst wurden für die Hypothesentestung drei Lichtbedingungen verwendet.

Die Probanden $(N=82)$ wurden randomisiert den Lichtbedingungen zugewiesen, jeweils zwei Personen zu einem Zeitpunkt. An der Studie nahmen insgesamt 53 Frauen und 29 Männer teil mit einem Durchschnittsalter von 22,28 $(S D=2,08)$ Jahren. Nach einer Expositionszeit von einer Stunde bewerteten die Probanden die Raumatmosphäre hinsichtlich Gemütlichkeit, Lebendigkeit und Distanziertheit [17]. Im Anschluss wurde den Probanden ein Szenario vorgegeben, das die Diskussion zwischen einem Bergsteiger (Rolle 1) und einem Finanzinvestor für eine Bergexpedition (Rolle 2) nachstellt [23]. Die zwei Verhandlungspartner verfolgten unterschiedliche Ziele (z. B. im Hinblick auf den Abreisetag), von welchen jedes Ziel mit einer Punktanzahl gewichtet war. Den Versuchsteilnehmern wurde zufällig die Rolle des Bergsteigers oder die des Finanzinvestors zugewiesen. Diese wurden aufgefordert, zusammen so viele Punkte wie möglich 
zu sammeln. Der Erfolg der Verhandlung wurde durch die gemeinsam erreichte Punktzahl in der Zweiergruppe $(\mathrm{N}=41)$ gemessen.
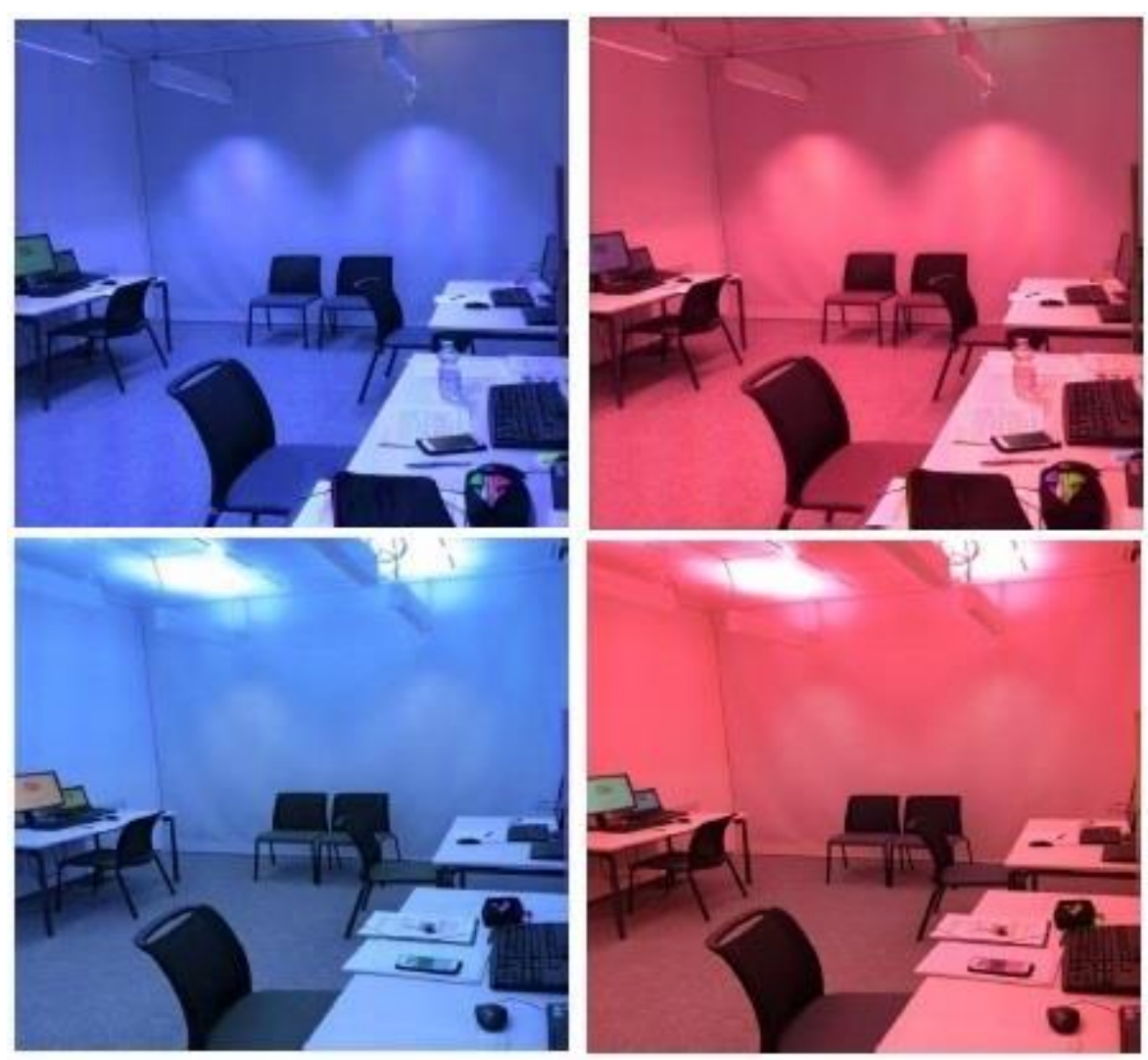

Bild 1. Die Lichtbedingungen mit dem farbigen Licht. Von links oben bis rechts unten: blau direkt, rot direkt, blau indirekt, rot indirekt.

\begin{tabular}{|c|c|c|c|c|c|}
\hline \multirow{2}{*}{$\begin{array}{l}\text { Lichtbe- } \\
\text { dingung }\end{array}$} & \multicolumn{2}{|c|}{$\begin{array}{l}\text { Durchschnittliche Be- } \\
\text { leuchtungsstärke (Lux) }\end{array}$} & \multirow{2}{*}{$\begin{array}{l}\text { Durchschnittli- } \\
\text { che Farbtempe- } \\
\text { ratur (Kelvin) }\end{array}$} & \multicolumn{2}{|c|}{ Koordinaten auf der CIE xy-Farbtafel } \\
\hline & horizontal & vertikal & & $\mathrm{X}$ & $\mathrm{y}$ \\
\hline Rot & 983 & 348 & 2156 & 0,434 & 0,296 \\
\hline Blau & 1244 & 415 & 18317 & 0,287 & 0,218 \\
\hline Weiß & 500 & 143 & 5597 & 0,332 & 0,329 \\
\hline
\end{tabular}

Tabelle 1. Die Lichtbedingungen in der experimentellen Studie

Wie erwartet bewerteten die Teilnehmer die Atmosphäre im Raum mit dem roten Licht als gemütlicher $(T(79)=2.53, p=.00, d=.40$, lebendiger $(T(79)=2.08, p=.02, d=.40$ und weni- 
ger distanziert $(T(79)=-4.53, p=.00, d=.40$ als in den anderen zwei Bedingungen (siehe Tabelle 2 für Mittelwerte). Außerdem erzielten die Teilnehmer in der Lichtsituation mit rotem Licht mehr Punkte bei der integrativen Verhandlung als in den anderen zwei Bedingungen ( $T$ (39) $=1.24, p=.05, d=.40$; siehe Tabelle 3 für Mittelwerte).

\begin{tabular}{|l|r|r|r|r|r|r|r|}
\hline Lichtfarbe & \multirow{2}{*}{$N$} & \multicolumn{2}{|c|}{ Gemütlichkeit } & \multicolumn{2}{|c|}{ Lebendigkeit } & \multicolumn{2}{c|}{ Distanziertheit } \\
\cline { 3 - 8 } & & Mittelwert & \multicolumn{1}{c|}{ SD } & Mittelwert & SD & Mittelwert & SD \\
\hline Rot & $33^{1}$ & 3.36 & 1.51 & 3.34 & 1.43 & 4.12 & 1.38 \\
\hline Blau & 32 & 2.45 & 1.18 & 2.73 & 1.31 & 5.34 & 1.12 \\
\hline Weiß & 16 & 2.98 & 0.89 & 2.77 & 0.85 & 5.30 & 0.79 \\
\hline
\end{tabular}

Tabelle 2. Lichtfarbe und die Raumatmosphäre. Aufgrund der a-priori Hypothese wurden einseitige T-Tests durchgeführt. ${ }^{1}$ Ein Teilnehmer hat die Fragen zu Raumatmosphäre nicht ausgefült.

\begin{tabular}{|l|c|c|c|}
\hline Lichtfarbe & $N$ (Dyade) & Mittelwert & $S D$ \\
\hline Rot & 17 & 13.58 & 0.42 \\
\hline Blau & 16 & 12.61 & 0.44 \\
\hline Weiß & 8 & 12.87 & 0.62 \\
\hline
\end{tabular}

Tabelle 3. Lichtfarbe und die erreichte Punktzahl in integrativer Verhandlung Notiz: aufgrund der a-priori Hypothese wurde der einseitige T-Test durchgeführt.

\section{Zusammenfassung}

Die vorliegende Studie untersuchte in einem experimentellen Ansatz, ob farbiges Licht, das eine visuelle Botschaft sozialer Wärme vermittelt, die Kooperation in integrativen Verhandlungen fördert. Die Ergebnisse unterstützen die Annahme, dass rotes Licht im Vergleich zu blauem und weißem Licht integrative Verhandlungen positiv beeinflusst.

Diese Studie leistet einen Beitrag zum Verständnis der Wirkung des farbigen Lichts. Im Gegensatz zu Objektfarbe kreiert die Lichtfarbe eine spezifische Raumatmosphäre, die Auswirkungen auf das Verhalten hat. Die Ergebnisse zeigen, dass rotes Licht eine gemütliche und informelle Atmosphäre erzeugt, die als passend für soziale Interaktionen empfunden werden kann. Diese Befunde gehen mit der früheren Forschung einher, die zeigt, dass physikalische Stimuli 
(bspw. warmes Licht) eine Basis für abstrakte psychologische Konstrukte bilden (bspw. interpersonale Wärme) und dadurch unser Verhalten beeinflussen [14]. Die Untersuchung des tatsächlichen kooperativen Verhaltens in einer integrativen Verhandlung erweitert frühere Befunde zur Wirkung des Lichts in sozialen Interaktionen [15, 18, 21].

Noch wichtiger ist, dass sich daraus praxisrelevante Implikationen ableiten lassen. Raumgestaltung soll demnach nicht nur nach vorgegebenen Normen stattfinden, sondern vielmehr den Menschen und seine Aktivitäten in den Fokus rücken. Eine optimal eingesetzte Lichtsituation kann nämlich eine Raumatmosphäre schaffen, die den Nutzer in seiner aktuellen Tätigkeit optimal unterstützt und eine Steigerung des gemeinschaftlichen Nutzens bewirkt. So zeigen die Ergebnisse der vorliegenden Studie, dass sozial warmes Licht den Erfolg einer integrativen Verhandlung positiv beeinflusst. Die Teilnehmer der experimentellen Studie erreichten die größte Punktzahl in der integrativen Verhandlung, wenn sie diese in einem rötlich beleuchteten Raum durchführten. Daraus lässt sich ableiten, dass in Räumen, in denen regelmäßig Verhandlungen stattfinden, das Anbieten alternativer Lichtszenarien von Vorteil sein kann. Die atmosphärischen Eigenschaften des Lichts sollen soziale Wärme und Einladung zu kooperativem Verhalten vermitteln, damit die integrative Verhandlung erfolgreich wird.

Die subjektiven Präferenzen im Hinblick auf die Lichtbedingungen sowie die persönliche Kontrolle sollten jedoch nicht außer Acht gelassen werden. Die Wichtigkeit der subjektiven Akzeptanz soll vor allem für das Wohlbefinden betont werden [24], das seinerseits Leistung und Verhalten beeinflusst [25]. Des Weiteren könnte der Effekt von Licht auf die Kooperation in Feldsituationen mit anderen Umweltfaktoren, wie z. B. Raumtemperatur, Luftqualität oder Geräuschkulisse, interagieren. Daher sollten weitere Felduntersuchungen die Replizierbarkeit der vorliegenden Ergebnisse in echten Verhandlungsräumen untersuchen. Hier müssen auch weitere Beleuchtungsaspekte wie unterschiedliche Tageslichteinflüsse betrachtet werden. Eine weitere Limitation stellt die kleine Stichprobe der experimentellen Untersuchung dar. Aus diesem Grund ist eine Replikation der Ergebnisse mit einer höheren Teilnehmeranzahl sinnvoll. Außerdem ist es wichtig anzumerken, dass die Farbwiedergabe abhängig vom Spektrum der installierten Leuchten war und es somit nicht möglich war bei der Farbwiedergabe in den farbigen Lichtbedingungen den empfohlenen Grenzwert von 80 [4] einzuhalten. Zukünftige Forschung könnte daher die Auswirkungen vom warmweißen und kaltweißen Licht, die die Mindestfarbwiedergabe von $R_{a}=80$ erreichen, auf Kooperation in Verhandlungen untersuchen.

Zusammengefasst lässt sich das Fazit ziehen, dass Licht das soziale Verhalten beeinflussen kann. Um Räume entsprechend, unterstützend für soziale Interaktionen, gestalten zu können, ist der Wissenstransfer von der Grundlagenforschung in die angewandte Forschung und in die Pra- 
xis nötig. So kann langfristig die Leistungsfähigkeit und das Wohlbefinden des Einzelnen durch die erfolgreiche Zusammenarbeit profitieren. Dies kann wiederum zum Erfolg der Organisationen beitragen.

\section{Danksagung}

Die Autoren danken dem Promotionskolleg „Menschen in Räumen“ und der Gips Schüle Stiftung für den fachlichen Austausch und die Unterstützung, die der Realisierung dieses Beitrags gedient haben. Vor allem möchten wir Herrn Dr.-Ing. Jan de Boer für seine hilfreichen Kommentare zu diesem Artikel danken. Des Weiteren bedanken wir uns bei Hannah Helfrich für das Korrekturlesen dieses Artikels und bei unseren studentischen Mitarbeitern für ihre Hilfe bei der Datenerhebung. 


\section{Literatur}

[1] Steidle, A., de Boer, J., Werth, L., \& Sedlbauer, K.: Stressor oder Ressource? Die Bedeutung der bauphysikalischen Bedingungen für den Menschen am Beispiel der Lichtumgebung [Stressor or resource? The significance of the physical conditions for individuals using the example of the luminous environment]. Bauphysik 5 (2015), pp. 263-267.

[2] Werth, L., Steidle, A., Hubschneider, C., de Boer, J. \& Sedlbauer, K.: Psychologische Befunde zu Licht und seiner Wirkung auf den Menschen - ein Überblick [Psychological evidence on light and its impact on humans - an overview]. Bauphysik 35 (2013), pp. 193-204.

[3] DIN 5034: Tageslicht in Innenräumen - Teil 1: Allgemeine Anforderungen. Berlin: Beuth Verlag 2011.

[4] DIN EN 12464-1: Licht und Beleuchtung - Beleuchtung von Arbeitsstätten - Teil 1: Arbeitsstätten in Innenräumen. Berlin: Beuth Verlag 2002

[5] Vischer, J. C.: The effects of the physical environment on job performance: Towards a theoretical model of workspace stress. Stress and Health 23 (2007), pp. 175-184.

[6] Kretschmer, V., Schmidt, K.-H., Griefahn, B.: Bright light effects on working memory, concentration and sustained attention of elderly night shift workers. Lighting Research and Technology (2011), pp. 1-18.

[7] Knez, I.: Effects of indoor lighting on mood and cognition. Journal of Environmental Psychology 15 (1995), pp. 39-51.

[8] Steidle, A., \& Werth, L.: Freedom from constraints: Darkness and dim illumination promote creativity. Journal of Environmental Psychology, 35 (2013), pp. 67-80.

[9] Boyce, P. R.: Lighting research for interiors: the beginning of the end or the end of the beginning. Lighting Research \& Technology 36 (2004), pp. 283-294.

[10]. Elliot, A.J., Maier, M.A., Moller, A.C., Friedmann, R., \& Meinhard, J.: Color and psychological functioning: The effect of red on performance attainment. Journal of Experimental Psychology: General 136 (2007), pp. 154-168

[11]. Maier, M.A., Elliot, A.J., \& Lichtenfeld, S.: Mediation of the negative effect of red on intellectual performance. Personality and Social Psychology Bulletin, 34 (2008), pp.1530-1540.

[12]. Elliot, A. J. \& Maier, M. A.: Color-in-context theory. In: Plant, A., Devine, P.: Advances in Experimental Social Psychology 45 (2012), pp. 61-125.

[13]. Rook, L.: Exposure to the color red enhances creative thinking depending on appetitiveaversive cues. Creativity Research Journal 26 (2014), pp. 124-130. 
[14]. Barsalou, L. W.: Grounded cognition. Annual Review of Psychology 59 (2008), pp. 617645.

[15] Kombeiz, O.: Turning the spotlight on the role of light and colors in offices: How are performance, social interactions, and social perception affected? Electronic published dissertation at University of Hohenheim (2016).

[16] Kuijsters, A., Redi, J., de Ruyter, B., \& Heynderickx, I.: Lighting to make you feelbetter: Improving the mood of elderly people with affective ambiences. PloS ONE, 10 (2015), pp. 122 .

[17] Vogels, I.M.L.C., de Vries M., \& van Erp, T.A.M.: Effect of coloured light on atmosphere perception. Proceedings of the AIC Interim Meeting, Swedish Colour Centre Foundation. Stockholm: AIC (2008), pp.15-18.

[18] Steidle, A., Hanke, E., \& Werth, L.: In the dark we cooperate: The situated nature of procedural embodiment. Social Cognition 31 (2013), pp. 275-300.

[19] Baron, R. A., Rea, M. S., \& Daniels, S. G.: Effects of indoor lighting (illuminance and spectral distribution) on the performance of cognitive tasks and interpersonal behaviors: The potential mediating role of positive affect. Motivation and Emotion 16 (1992), pp. 1-33.

[20] Knez, I.: Effects of indoor lighting on mood and cognition. Journal of Environmental Psychology 15 (1995), pp. 39-51.

[21] Takahashi, S.: Effects of coloured lighting on judgement of emotional expression. Journal of the International Colour Association 4 (2009), pp. 1-9.

[22] Carnevale, P. J., \& Isen, A. M. The influence of positive affect and visual access on the discovery of integrative solutions in bilateral negotiation. Organizational Behavior and Human Decision Processes 37 (1986), pp. 1-13.

[23] Gockel, C. Integrative Verhandlung für Konflikt- und Verhandlungstraining. Unveröffentlichte Version (2014).

[24] Veitch, J. A., Newsham, G. R., Boyce, P. R., \& Jones, C. C.: Lighting appraisal, well-being and performance in open-plan offices: A linked mechanisms approach. Lighting Research and Technology 40 (2008), pp. 133-151.

[25] Wright, T. A., \& Cropanzano, R.: Psychological well-being and job satisfaction as predictors of job performance. Journal of occupational health psychology 5 (2000), pp. 84-94. 